\title{
Benign Retroperitoneal Neoplasm
}

National Cancer Institute

\section{Source}

National Cancer Institute. Benign Retroperitoneal Neoplasm. NCI Thesaurus. Code C4447.

A non-metastasizing neoplasm that arises from the retroperitoneum. 